\title{
Crescimento e esporulação de Bipolaris euphorbiae cultivado sob diferentes condições nutricionais
}

\author{
Growth and sporulation of Bipolaris euphorbiae cultivated under different nutritional conditions
}

\author{
Mara Cristina Penariol ${ }^{\mathrm{I}}$ Antonio Carlos Monteiro ${ }^{\mathrm{II}}$ Robinson Antonio PitelliII
}

\section{RESUMO}

Apesar de ser considerado um potencial bioagente de controle do amendoim-bravo (Euphorbia heterophylla L.) em cultivos de soja no Brasil, pouco se conhece sobre as características nutricionais do fungo Bipolaris euphorbiae Muchovej \& Carvalho. O objetivo deste trabalho foi avaliar diferentes fontes de carbono (glicose, amido, sacarose, lactose e maltose), de nitrogênio (nitrato de sódio, nitrato de amônio, sulfato de amônio, fosfato de amônio dibásico, cloreto de amônio), de fósforo (fosfato de potássio monobásico, fosfato de potássio bibásico, fosfato de cálcio, fosfato de sódio, ácido fosfórico, fosfato de amônio) e a suplementação do meio de cultivo com fontes orgânicas de macro e micronutrientes, para o crescimento e a esporulação de B. euphorbiae. O amido proporcionou o maior crescimento, sendo também considerada a fonte de carbono mais favorável para a esporulação. $O$ melhor crescimento e esporulação de B. euphorbiae foram obtidos usando o nitrato de sódio como fonte de nitrogênio $e$ fosfato de potássio monobásico e fosfato de cálcio como fonte de fósforo, embora as demais fontes de fósforo analisadas, exceto o ácido fosfórico, tenham igualmente favorecido a esporulação. A suplementação do meio de cultivo com peptona e extrato de levedura resultou em melhor crescimento $e$ esporulação do fungo e a adição de vitaminas favoreceu a produção de conídios.

Palavras-chave: planta daninha, controle biológico, meio de cultura, suplementos.

\section{ABSTRACT}

Bipolaris euphorbiae Muchovej \& Carvalho is considered a potential bioagent for control of selvage peanuts (Euphorbia heterophylla L.) on soybean crops in Brazil.
However, little is known about the nutritional characteristics of this fungus. The main objective of this research was to evaluate different sources of carbon (glucose, starch, sucrose, lactose and maltose), of nitrogen (sodium nitrate, ammonium nitrate, ammonium sulphate, bi-basic ammonium phosphate, ammonium chloride), of phosphorus (monobasic potassium phosphate, bi-basic potassium phosphate, calcium phosphate, sodium phosphate, phosphoric acid, ammonium phosphate) and addition of organic sources of macro and micronutrients as supplement in the culture medium for the growth and sporulation of $\boldsymbol{B}$. euphorbiae. Starch was the carbon source that promoted the greater fungus growth and was considered the most favorable to sporulation. The nitrogen source sodium nitrate and the phosphorus source monobasic potassium phosphate and calcium phosphate provided the best growth and sporulation of $\boldsymbol{B}$. euphorbiae, but the others phosphorus source analyzed, except phosphoric acid, favored the fungus sporulation. The supplementation of the culture medium with peptone and yeast extract promoted a better growth and sporulation of the fungus and the addition of vitamins favored the production of conidia.

Key words: weed, biological control, culture medium, supplements.

\section{INTRODUÇÃO}

Euphorbia heterophylla, conhecida popularmente como amendoim-bravo ou leiteiro, é uma planta daninha amplamente distribuída no Brasil, principalmente nas regiões onde se cultiva a soja (OLIVEIRA \& SÁ, 1998). Em função do rápido

\footnotetext{
'Programa de Pós-graduação em Microbiologia Agropecuária, Faculdade de Ciências Agrárias e Veterinárias (FCAV), Universidade Estadual Paulista (Unesp), Jaboticabal, SP, Brasil.

IDepartamento de Produção Vegetal, FCAV, Unesp. Via de Acesso Prof. Paulo Donato Castellane, s/n, 14884-900, Jaboticabal, SP, Brasil. E-mail: montecar@fcav.unesp.br. Autor para correspondência.

IIIDepartamento de Biologia Aplicada a Agropecuária, FCAV, Unesp, Jaboticabal, SP, Brasil.
} 
crescimento inicial, a planta exerce forte competição por luz, nutrientes do solo e água, além de produzir grande quantidade de sementes que germinam numa ampla faixa de condições ambientais (ETEJERE \& OKOKO, 1989). O controle químico é efetuado principalmente pelo uso de inibidores da enzima acetolase sintetase (ALS), mas são observadas falhas devido ao aparecimento de biótipos resistentes a esses herbicidas (CHRISTOFFOLETTI et al., 1994).

O emprego de bioherbicidas, entre os quais o fungo Bipolaris euphorbiae, tem sido sugerido como estratégia para o controle biológico de $\boldsymbol{E}$. heterophylla. O fungo é patógeno específico para $\boldsymbol{E}$. heterophylla, provocando manchas necróticas no caule e nas folhas, evoluindo para intenso desfolhamento, podendo conduzir à sua morte. Nas condições em que não ocorre a morte da planta, sua capacidade competitiva fica drasticamente reduzida (YORINORI, 1985). Em aplicações concentradas de suspensão de esporos, GAZZIERO \& YORINORI (1993) verificaram que $\boldsymbol{B}$. euphorbiae provocou intenso desfolhamento e lesões caulinares em E. heterophylla. Por ser de fácil esporulação, o patógeno foi considerado um candidato ideal para o desenvolvimento de bioherbicida a ser introduzido em programas de manejo do amendoimbravo.

Um dos entraves para utilização de fungos como bioagentes de controle de plantas daninhas reside na produção de inóculo em grandes quantidades, sem perda de virulência. Mesmo quando se dispõe de uma técnica eficiente de propagação de inóculo, é necessário encontrar substratos que permitam produzir o fungo em larga escala e que tornem o produto economicamente viável. Para tanto, é importante conhecer as condições de cultivo que permitam obter bom crescimento do patógeno com alta esporulação, fazendo-se necessário investigar sua habilidade em usar diferentes fontes de carbono, nitrogênio e fósforo, e suas necessidades em termos de outros macro ou micronutrientes e fatores de crescimento.

$O$ fungo $\boldsymbol{B}$. euphorbiae cresceu em temperaturas variando entre 10 e $30^{\circ} \mathrm{C}$, com valor ótimo em $25^{\circ} \mathrm{C}$, e a melhor germinação de conídios ocorreu no intervalo entre 20 e $30^{\circ} \mathrm{C}$ (NECHET et al., 2006), enquanto a melhor taxa de crescimento de Bipolaris sorokiniana (Sacc. in Sorok) foi obtida entre 26 e $28^{\circ} \mathrm{C}$ (PRATES \& FERNANDES, 2000). Alternaria brassicae (Berk.) Sacc. e Alternaria raphani Groves e Skollo cresceram rapidamente quando foi adicionado amido ao meio de cultura, e Alternaria brassicicola (Schw.) Wiltshire cresceu melhor em meio contendo galactose (TABER et al., 1968). A produção de $5 \times 10^{3}$ conídios $\mathrm{mL}^{-1}$ e $4 \times 10^{3}$ conídios $\mathrm{mL}^{-1}$ de Alternaria alternata
(Fr.) Keissler foi obtida usando galactose e arabinose, respectivamente, como fonte de carbono, no entanto, não foram verificadas diferenças em relação às fontes de nitrogênio avaliadas (SILVA \& MELO, 1999).

Trabalhos relativos a análises das exigências nutricionais de B. euphorbiae não foram encontrados na literatura. Assim, este trabalho teve por objetivo avaliar diferentes fontes de carbono, nitrogênio, fósforo e a suplementação do meio de cultivo com fontes orgânicas de macro e micronutrientes, para o crescimento e a esporulação de B. euphorbiae.

\section{MATERIAL E MÉTODOS}

Neste estudo foi utilizado o isolado FCAV\# 569 de B. euphorbiae, cedido pelo Laboratório de Controle Biológico de Plantas Daninhas "Prof. Giorgio de Marinis" da Faculdade de Ciências Agrárias e Veterinárias, Unesp, Câmpus de Jaboticabal, SP. O isolado permaneceu estocado em tubos de ensaio mantidos a $4{ }^{\circ} \mathrm{C}$ contendo meio de batata-dextroseágar (BDA). Para utilização nos ensaios, o fungo foi cultivado em placas de Petri contendo o Meio Mínimo de Pontecorvo (PONTECORVO et al., 1953), com a seguinte composição: $6,0 \mathrm{~g}$ de $\mathrm{NaNO}_{3} ; 1,52 \mathrm{~g}$ de $\mathrm{KH}_{2} \mathrm{PO}_{4} ; 0,52 \mathrm{~g}$ de $\mathrm{MgSO}_{4} .7 \mathrm{H}_{2} \mathrm{O} ; 0,52 \mathrm{~g}$ de $\mathrm{KCl} ; 0,01 \mathrm{~g}$ de $\mathrm{FeSO}_{4} ; 0,01 \mathrm{~g}$ de $\mathrm{ZnCl}_{4} ; 10 \mathrm{~g}$ de glicose; $15 \mathrm{~g}$ de ágar e $1000 \mathrm{~mL}$ de água destilada. Após esterilização em autoclave a $121^{\circ} \mathrm{C}$ e $1 \mathrm{Kgf} \mathrm{cm}^{-2}$, por 20 minutos, foi vertido em placas de Petri que foram mantidas em BOD a $27^{\circ} \mathrm{C}$ por 24 horas para secagem da superfície do meio, evitando que a umidade excessiva pudesse espalhar propágulos do fungo após a inoculação. Discos de $5 \mathrm{~mm}$ de diâmetro, contendo micélio e esporos obtidos de colônias jovens do fungo, foram transferidos com o auxílio da uma alça de níquel-cromo estéril para o centro de cada placa de Petri. As placas foram mantidas em $\mathrm{BOD} \mathrm{a} 27^{\circ} \mathrm{C}$ por 21 dias, na ausência de iluminação.

Foram organizados quatro ensaios. Em todos foi utilizado o meio mínimo como meio base, foram avaliadas a fonte nutricional em análise. No primeiro ensaio, avaliaram-se diferentes fontes de carbono (glicose, sacarose, maltose, lactose e amido), acrescidas ao meio na quantidade de $10 \mathrm{~g} \mathrm{~L}^{-1}$. No segundo, o fungo foi cultivado em meio contendo diferentes fontes de nitrogênio [nitrato de sódio $\left(\mathrm{NaNO}_{3}\right)$, nitrato de amônio $\left(\mathrm{NH}_{4} \mathrm{NO}_{3}\right)$, sulfato de amônio $\left[\left(\mathrm{NH}_{4}\right) \mathrm{H}_{2} \mathrm{SO}_{4}\right]$, fosfato de amônio dibásico $\left[\left(\mathrm{NH}_{4}\right)_{2} \mathrm{HPO}_{4}\right]$, cloreto de amônio $\left.\left(\mathrm{NH}_{4} \mathrm{Cl}\right)\right]$, adicionadas na concentração de $6,0 \mathrm{~g} \mathrm{~L}^{-1}$ e a fonte de carbono usada foi a que proporcionou o melhor desempenho do fungo no ensaio anterior. 
No terceiro ensaio, foi examinada a influência de diferentes fontes de fósforo [fosfato de potássio monobásico $\left(\mathrm{KH}_{2} \mathrm{PO}_{4}\right)$, fosfato de potássio bibásico $\left(\mathrm{K}_{2} \mathrm{HPO}_{4}\right)$, fosfato de cálcio $\left(\mathrm{CaHPO}_{4}\right)$, fosfato de sódio $\left(\mathrm{NaH}_{2} \mathrm{PO}_{4}\right)$, ácido fosfórico $\left(\mathrm{H}_{3} \mathrm{PO}_{4}\right)$ e fosfato de amônio dibásico $\left.\left(\mathrm{NH}_{4}\right)_{2} \mathrm{HPO}_{4}\right]$. Cada fonte foi adicionada ao meio na concentração de $1,52 \mathrm{~g} \mathrm{~L}^{-1} \mathrm{e}$ as fontes de carbono e nitrogênio utilizadas foram as que proporcionaram melhor desempenho do fungo nos ensaios anteriores. No último ensaio, foi analisado o efeito da suplementação do meio de cultivo adicionando-se ao meio mínimo, elaborado com as fontes de carbono, nitrogênio e fósforo que proporcionaram melhor desempenho do fungo nos ensaios anteriores, os demais nutrientes usados na composição do Meio Completo de Pontecorvo (Pontecorvo et al., 1953, modificado por AZEVEDO \& COSTA, 1973), com exceção do hidrolisado de ácido nucléico de levedura. Os tratamentos do ensaio foram: meio mínimo adicionado de $2,0 \mathrm{~g}$ de peptona; meio mínimo adicionado de $1,5 \mathrm{~g}$ de caseína hidrolisada; meio mínimo adicionado de $0,5 \mathrm{~g}$ de extrato de levedura; meio mínimo adicionado de $1,0 \mathrm{~mL}$ de solução de vitaminas; meio mínimo adicionado de todos os ingredientes anteriores, que corresponde ao Meio Completo de Pontecorvo.

A solução de vitaminas foi assim composta: $0,2 \mathrm{mg}$ de biotina; $10,0 \mathrm{mg}$ de ácido $\beta$ - aminobenzóico; $50,0 \mathrm{mg}$ de tiamina; $50,0 \mathrm{mg}$ de piridoxina; $100,0 \mathrm{mg}$ de ácido nicotínico; $100,0 \mathrm{mg}$ de riboflavina; $100,0 \mathrm{~mL}$ de água destilada. A solução foi submetida à esterilização fracionada e conservada em frasco escuro no refrigerador a $4^{\circ} \mathrm{C}$, sob clorofórmio.

Em todos os ensaios foi avaliado o crescimento radial das colônias de B. euphorbiae, medindo-se a cada três dias, durante 21 dias, com auxílio de régua milimétrica, quatro eixos ortogonais previamente marcados na face externa de cada placa. Após incubação por 21 dias a $27^{\circ} \mathrm{C}$, a superfície de cada colônia foi cuidadosamente raspada com espátula e o conteúdo foi transferido individualmente para tubos contendo $10 \mathrm{~mL}$ de uma mistura $(1: 1)$ de solução salina $(\mathrm{NaCl}$ a $0,89 \%)$ e Tween $80(0,1 \%)$. Após vigorosa agitação em agitador elétrico de tubos, a quantidade de esporos da suspensão foi determinada em câmara de Neubauer, usando diluição da suspensão se necessário.

Os dados de crescimento foram analisados segundo o delineamento inteiramente casualizado e os valores médios foram comparados pelo teste de Tukey $(\mathrm{P}>0,05)$. Os dados referentes à esporulação foram transformados em $\log (\mathrm{x}+1)$ e submetidos à análise de variância pelo teste $\mathrm{F}$, sendo as médias comparadas pelo teste de Tukey a 5\% de probabilidade. Para execução das análises foi utilizado o programa SAS (1995). Tanto para a avaliação de crescimento como da esporulação foram feitas cinco repetições por tratamento.

\section{RESULTADOS E DISCUSSÃO}

$\mathrm{O}$ amido foi a fonte de carbono que promoveu maior crescimento da colônia do fungo (Tabela 1), constituindo-se na fonte mais adequada, pois, com apenas nove dias de cultivo, as hifas de $\boldsymbol{B}$. euphorbiae quase haviam atingido a borda da placa de Petri, fato que limitou o crescimento a partir de então. Até o nono dia de cultivo o crescimento do fungo obtido em presença de maltose, sacarose e glicose não diferiu estatisticamente. A partir, do $12^{\circ}$ dia, iniciou-se diferenciação no crescimento, destacando-se maltose e sacarose como as fontes mais promissoras, e no $21^{\circ}$ de cultivo, sacarose promoveu maior crescimento do fungo.

Não foi verificada diferença estatística na produção de conídios de $\boldsymbol{B}$. euphorbiae obtida nos tratamentos contendo amido, sacarose, glicose, maltose e lactose (Tabela 2), mas o amido é, provavelmente, a fonte mais favorável, pois estimulou acentuadamente a esporulação. Esse fato, aliado aos resultados de crescimento, sugere que $\boldsymbol{B}$. euphorbiae tenha boa capacidade de desdobrar amido como fonte de carbono, produzindo diversas amilases.

Segundo CARLILE \& WATKINSON (1994), muitos fungos utilizam glicose como fonte de carbono, porém, vários outros açúcares podem ser aproveitados. $\mathrm{O}$ amido foi a fonte de carbono que proporcionou crescimento mais rápido de $A$. brassicae e A. raphani (TABER et al, 1968). Helminthoporium orysae Breda de Haan pode utilizar frutose, arabinose, sacarose, maltose e amido como fontes de carbono (DIAZ, 1995). MONTEIRO (1988) verificou que glicose e amido favoreceram o crescimento de Metarhizium anisopliae (Metsch.) Sorok. e Beauveria bassiana (Bals.) Vuill., enquanto glicose e sacarose foram as fontes de carbono mais adequadas para o crescimento e a esporulação de Paecilomyces marquandii (Masse) Hughes. Glicose, manitol e sacarose foram as melhores fontes de carbono para crescimento e esporulação de Verticillium fungicola (Pruess) Hassebrauk (COETZEE \& EICKER, 1991). BARBOSA et al. (2002) verificaram que o melhor crescimento e a esporulação do isolado JAB 02 de Verticillium lecanii (=Lecanicillium lecanii Zare e Gams) foram obtidos em meio contendo lactose e, para JAB 45, o meio contendo glicose foi o mais favorável. 
Tabela 1 - Crescimento radial médio (mm) de Bipolaris euphorbiae após cultivo por 21 dias a $27^{\circ} \mathrm{C}$ e em ausência de iluminação, em meio mínimo contendo diferentes fontes de carbono, nitrogênio, fósforo e suplementos.

\begin{tabular}{|c|c|c|c|c|c|c|c|}
\hline \multirow{2}{*}{ Fator avaliado } & \multicolumn{7}{|c|}{ - } \\
\hline & 3 & 6 & 9 & 12 & 15 & 18 & 21 \\
\hline \multicolumn{8}{|l|}{ Fonte de carbono } \\
\hline Maltose & $16,0 \mathrm{~B}$ & $22,8 \mathrm{~B}$ & $32,6 \mathrm{~B}$ & $42,2 \mathrm{~B}$ & $49,2 \mathrm{~B}$ & $53,4 \mathrm{~B}$ & $54,0 \mathrm{C}$ \\
\hline Lactose & $13,6 \mathrm{~B}$ & $18,0 \mathrm{C}$ & $24,8 \mathrm{C}$ & $31,2 \mathrm{D}$ & $37,2 \mathrm{C}$ & $43,6 \mathrm{C}$ & $52,6 \mathrm{C}$ \\
\hline Sacarose & $12,8 \mathrm{~B}$ & $20,0 \mathrm{BC}$ & $28,8 \mathrm{BC}$ & $40,0 \mathrm{BC}$ & $49,8 \mathrm{~B}$ & $57,2 \mathrm{~B}$ & $59,4 \mathrm{~B}$ \\
\hline Glicose & $14,4 \mathrm{~B}$ & $21,4 \mathrm{BC}$ & $27,6 \mathrm{BC}$ & $34,4 \mathrm{CD}$ & $41,4 \mathrm{C}$ & $47,4 \mathrm{C}$ & $51,4 \mathrm{C}$ \\
\hline Amido & $33,0 \mathrm{~A}$ & $62,0 \mathrm{~A}$ & $88,6 \mathrm{~A}$ & $90,0 \mathrm{~A}$ & $90,0 \mathrm{~A}$ & $90,0 \mathrm{~A}$ & $90,0 \mathrm{~A}$ \\
\hline Média + Erro padrão & $18,0 \pm 1,6$ & $28,8 \pm 3,4$ & $40,5 \pm 5,0$ & $47,6 \pm 4,4$ & $53,5 \pm 3,9$ & $58,3 \pm 3,4$ & $61,5 \pm 3,0$ \\
\hline CV $(\%)$ & 10,9 & 7,6 & 9,3 & 7,6 & 6,2 & 4,3 & 3,9 \\
\hline \multicolumn{8}{|l|}{ Fonte de nitrogênio ${ }^{1}$} \\
\hline $\mathrm{NaNO}_{3}$ & $28,8 \mathrm{~A}$ & $76,8 \mathrm{~A}$ & $90,0 \mathrm{~A}$ & $90,0 \mathrm{~A}$ & $90,0 \mathrm{~A}$ & $90,0 \mathrm{~A}$ & $90,0 \mathrm{~A}$ \\
\hline$\left(\mathrm{NH}_{4}\right)_{2} \mathrm{HPO}_{4}$ & $16,2 \mathrm{C}$ & $30,0 \mathrm{~B}$ & $46,5 \mathrm{~B}$ & $62,9 \mathrm{~B}$ & $77,5 \mathrm{~B}$ & $86,6 \mathrm{~B}$ & $90,0 \mathrm{~A}$ \\
\hline $\mathrm{NH}_{4} \mathrm{Cl}$ & $13,0 \mathrm{D}$ & $18,2 \mathrm{C}$ & $20,0 \mathrm{D}$ & $21,2 \mathrm{D}$ & $22,6 \mathrm{D}$ & $22,6 \mathrm{D}$ & $23,0 \mathrm{C}$ \\
\hline$\left(\mathrm{NH}_{4}\right)_{2} \mathrm{SO}_{4}$ & $13,3 \mathrm{D}$ & $19,0 \mathrm{C}$ & $19,0 \mathrm{D}$ & $20,8 \mathrm{D}$ & $20,9 \mathrm{D}$ & $21,3 \mathrm{D}$ & $21,5 \mathrm{C}$ \\
\hline $\mathrm{NH}_{4} \mathrm{NO}_{3}$ & $20,0 \mathrm{~B}$ & $33,1 \mathrm{~B}$ & $33,1 \mathrm{C}$ & $33,1 \mathrm{C}$ & $33,1 \mathrm{C}$ & $33,2 \mathrm{C}$ & $33,3 \mathrm{~B}$ \\
\hline Média + Erro padrão & $18,3 \pm 1,2$ & $35,4 \pm 4,4$ & $41,7 \pm 5,3$ & $45,6 \pm 5,5$ & $48,8 \pm 5,9$ & $50,7 \pm 6,3$ & $51,6 \pm 6,5$ \\
\hline $\mathrm{CV}(\%)$ & 6,7 & 5,7 & 3,2 & 2,9 & 3,4 & 3,0 & 3,1 \\
\hline \multicolumn{8}{|l|}{ Fonte de fósforo ${ }^{2}$} \\
\hline $\mathrm{KH}_{2} \mathrm{PO}_{4}$ & $23,8 \mathrm{~A}$ & $62,3 \mathrm{~B}$ & $90,0 \mathrm{~A}$ & $90,0 \mathrm{~A}$ & $90,0 \mathrm{~A}$ & $90,0 \mathrm{~A}$ & $90,0 \mathrm{~A}$ \\
\hline $\mathrm{K}_{2} \mathrm{HPO}_{4}$ & $18,5 \mathrm{~B}$ & $41,8 \mathrm{C}$ & $67,2 \mathrm{C}$ & $80,2 \mathrm{~B}$ & $90,0 \mathrm{~A}$ & $90,0 \mathrm{~A}$ & $90,0 \mathrm{~A}$ \\
\hline $\mathrm{CaHPO}_{4}$ & $26,7 \mathrm{~A}$ & $73,4 \mathrm{~A}$ & $90,0 \mathrm{~A}$ & $90,0 \mathrm{~A}$ & $90,0 \mathrm{~A}$ & $90,0 \mathrm{~A}$ & $90,0 \mathrm{~A}$ \\
\hline $\mathrm{NaH}_{2} \mathrm{PO}_{4}$ & $19,3 \mathrm{~B}$ & $45,5 \mathrm{C}$ & $71,9 \mathrm{~B}$ & $90,0 \mathrm{~A}$ & $90,0 \mathrm{~A}$ & $90,0 \mathrm{~A}$ & $90,0 \mathrm{~A}$ \\
\hline$\left(\mathrm{NH}_{4}\right)_{2} \mathrm{HPO}_{4}$ & $18,8 \mathrm{~B}$ & $43,8 \mathrm{C}$ & $66,8 \mathrm{C}$ & $79,6 \mathrm{~B}$ & $83,5 \mathrm{~B}$ & $86,2 \mathrm{~B}$ & $90,0 \mathrm{~A}$ \\
\hline $\mathrm{H}_{3} \mathrm{PO}_{4}$ & $5,0 \mathrm{C}$ & $5,0 \mathrm{D}$ & $12,1 \mathrm{D}$ & $17,7 \mathrm{C}$ & $23,2 \mathrm{C}$ & $33,2 \mathrm{C}$ & $39,3 \mathrm{~B}$ \\
\hline Média + Erro padrão & $18,7 \pm 1,4$ & $45,3 \pm 4,5$ & $66,3 \pm 5,3$ & $74,6 \pm 5,6$ & $77,8 \pm 5,3$ & $79,9 \pm 4,6$ & $81,5 \pm 4,1$ \\
\hline CV $(\%)$ & 11,0 & 4,3 & 3,2 & 0,9 & 1,6 & 1,4 & 0,7 \\
\hline \multicolumn{8}{|l|}{ Suplemento $^{3}$} \\
\hline Meio mínimo & $23,5 \mathrm{~B}$ & 76,4 B & $90,0 \mathrm{~A}$ & $90,0 \mathrm{~A}$ & $90,0 \mathrm{~A}$ & $90,0 \mathrm{~A}$ & $90,0 \mathrm{~A}$ \\
\hline MM + E.L. & $32,9 \mathrm{AB}$ & $84,5 \mathrm{AB}$ & $90,0 \mathrm{~A}$ & $90,0 \mathrm{~A}$ & $90,0 \mathrm{~A}$ & $90,0 \mathrm{~A}$ & $90,0 \mathrm{~A}$ \\
\hline MM + Caseína & $32,8 \mathrm{AB}$ & $76,0 \mathrm{~B}$ & $90,0 \mathrm{~A}$ & $90,0 \mathrm{~A}$ & $90,0 \mathrm{~A}$ & $90,0 \mathrm{~A}$ & $90,0 \mathrm{~A}$ \\
\hline MM + Vitamina & $24,0 \mathrm{~B}$ & $81,2 \mathrm{AB}$ & $90,0 \mathrm{~A}$ & $90,0 \mathrm{~A}$ & $90,0 \mathrm{~A}$ & $90,0 \mathrm{~A}$ & $90,0 \mathrm{~A}$ \\
\hline MM + Peptona & $31,8 \mathrm{AB}$ & $85,9 \mathrm{~A}$ & $90,0 \mathrm{~A}$ & $90,0 \mathrm{~A}$ & $90,0 \mathrm{~A}$ & $90,0 \mathrm{~A}$ & $90,0 \mathrm{~A}$ \\
\hline Meio completo & $36,4 \mathrm{~A}$ & $87,9 \mathrm{~A}$ & $90,0 \mathrm{~A}$ & $90,0 \mathrm{~A}$ & $90,0 \mathrm{~A}$ & $90,0 \mathrm{~A}$ & $90,0 \mathrm{~A}$ \\
\hline Média + Erro padrão & $30,2 \pm 1,4$ & $82,0 \pm 1,3$ & $90,0 \pm 0,0$ & $90,0 \pm 0,0$ & $90,0 \pm 0,0$ & $90,0 \pm 0,0$ & $90,0 \pm 0,0$ \\
\hline CV $(\%)$ & 16,1 & 5,8 & - & - & - & - & - \\
\hline
\end{tabular}

Médias seguidas pela mesma letra, nas colunas de cada fator avaliado, não diferem entre si pelo teste de Tukey a 5\% de probabilidade.

${ }^{1}$ Meio de cultura contendo amido como fonte de carbono.

${ }^{2}$ Meio de cultura contendo amido e nitrato de sódio como fontes de carbono e nitrogênio, respectivamente.

${ }^{3}$ Meio de cultura contendo amido, nitrato de sódio e fosfato de potássio monobásico, como fontes de carbono, nitrogênio e fósforo, respectivamente.

MM: meio mínimo; EL: extrato de levedura.

O crescimento de B. euphorbiae foi maior em meio contendo nitrato de sódio como fonte de nitrogênio (Tabela 1). Nesse tratamento, o fungo colonizou toda a área disponível já no nono dia de cultivo e, a partir daí, o crescimento foi limitado pelo diâmetro da placa. O meio contendo fosfato de amônio 
Tabela 2 - Esporulação de Bipolaris euphorbiae após cultivo por 21 dias a $27^{\circ} \mathrm{C}$ e em ausência de iluminação, em meio mínimo contendo diferentes fontes de carbono nitrogênio, fósforo e suplementos.

\begin{tabular}{|c|c|}
\hline Fator avaliado & Número de conídios $\mathrm{x} 10^{5}$ \\
\hline \multicolumn{2}{|l|}{ Fonte de carbono } \\
\hline Maltose & $52,3 \pm 5,3 \mathrm{~A}$ \\
\hline Lactose & $26,6 \pm 7,4 \mathrm{~A}$ \\
\hline Sacarose & $76,0 \pm 15,4 \mathrm{~A}$ \\
\hline Glicose & $84,5 \pm 15,9 \mathrm{~A}$ \\
\hline Amido & $195,0 \pm 120,9 \mathrm{~A}$ \\
\hline $\mathrm{CV}(\%)$ & 20,6 \\
\hline \multicolumn{2}{|c|}{ Fonte de nitrogênio ${ }^{1}$} \\
\hline $\mathrm{NaNO}_{3}$ & $190,0 \pm 34,8 \mathrm{~A}$ \\
\hline$\left(\mathrm{NH}_{4}\right)_{2} \mathrm{HPO}_{4}$ & $44,2 \pm 11,1 \mathrm{~B}$ \\
\hline $\mathrm{NH}_{4} \mathrm{Cl}$ & $1,4 \pm 1,1 \mathrm{C}$ \\
\hline$\left(\mathrm{NH}_{4}\right)_{2} \mathrm{SO}_{4}$ & $3,9 \pm 1,6 \mathrm{C}$ \\
\hline $\mathrm{NH}_{4} \mathrm{NO}_{3}$ & $0,3 \pm 0,3 \mathrm{C}$ \\
\hline $\mathrm{CV}(\%)$ & 30,7 \\
\hline \multicolumn{2}{|l|}{ Fonte de fósforo ${ }^{2}$} \\
\hline $\mathrm{KH}_{2} \mathrm{PO}_{4}$ & $290,7 \pm 62,0 \mathrm{~A}$ \\
\hline $\mathrm{K}_{2} \mathrm{HPO}_{4}$ & $241,3 \pm 24,9 \mathrm{~A}$ \\
\hline $\mathrm{CaHPO}_{4}$ & $202,0 \pm 70,3 \mathrm{~A}$ \\
\hline $\mathrm{NaH}_{2} \mathrm{PO}_{4}$ & $188,1 \pm 20,7 \mathrm{~A}$ \\
\hline$\left(\mathrm{NH}_{4}\right)_{2} \mathrm{HPO}_{4}$ & $132,0 \pm 13,4 \mathrm{~A}$ \\
\hline $\mathrm{H}_{3} \mathrm{PO}_{4}$ & $0,4 \pm 0,2 \mathrm{~B}$ \\
\hline $\mathrm{CV}(\%)$ & 0,05 \\
\hline \multicolumn{2}{|l|}{ Suplemento $^{3}$} \\
\hline Meio mínimo & $202,2 \pm 30,0 \mathrm{~B}$ \\
\hline MM + E.L. & $508,0 \pm 97,9 \mathrm{AB}$ \\
\hline MM + Caseína & $303,7 \pm 96,0 \mathrm{~B}$ \\
\hline MM + Vitamina & $431,0 \pm 42,0 \mathrm{AB}$ \\
\hline MM + Peptona & $719,4 \pm 141,7 \mathrm{~A}$ \\
\hline Meio completo & $734,0 \pm 87,8 \mathrm{~A}$ \\
\hline $\mathrm{CV}(\%)$ & 6,15 \\
\hline
\end{tabular}

Valores médios originais seguidos do erro padrão da média, mas análise estatística realizada com dados transformados em log $(\mathrm{x}+$ $1)$.

Médias seguidas pela mesma letra, na coluna de cada fator avaliado, não diferem entre si pelo teste de Tukey a $5 \%$ de probabilidade.

${ }^{1}$ Meio de cultura contendo amido como fonte de carbono.

${ }^{2}$ Meio de cultura contendo amido e nitrato de sódio como fontes de carbono e nitrogênio, respectivamente.

${ }^{3}$ Meio de cultura contendo amido, nitrato de sódio e fosfato de potássio monobásico, como fontes de carbono, nitrogênio e fósforo, respectivamente.

dibásico também favoreceu o crescimento de $\boldsymbol{B}$. euphorbiae, mas o mesmo efeito ocorrido com nitrato de sódio só foi verificado no $21^{\circ}$ dia de incubação. Quando foram utilizadas as fontes cloreto de amônio, sulfato de amônio e nitrato de amônio foi obtido menor crescimento do fungo, o que ficou evidente a partir do nono dia de incubação.

O nitrato de sódio foi também a fonte que melhor promoveu a esporulação de B. euphorbiae (Tabela 2), diferindo das produções de conídios obtidas nos meios com as demais fontes. Na presença de sulfato de amônio, cloreto de amônio e nitrato de amônio a esporulação do fungo foi acentuadamente menor.

Os resultados mostraram que B. euphorbiae teve maior capacidade de utilizar nitrato de sódio que os sais que contém o ion amônio como fonte de nitrogênio. Esse fato sugere que o fungo tenha habilidade de sintetizar enzimas envolvidas no mecanismo de redução do nitrato, como nitrato redutase, nitrito redutase e hidroxiamino redutase (GARRAWAY \& EVANS, 1984). Segundo esses autores, o nitrogênio é indispensável para o desenvolvimento de fungos, sendo necessário para a síntese de componentes celulares importantes, tais como aminoácidos, ácidos nucléicos e quitina. BARBOSA et al. (2002) encontraram que nitrato de sódio e fosfato de amônio favoreceram o desenvolvimento de V. lecanii.

B. euphorbiae cresceu rapidamente na presença de fosfato de potássio monobásico e fosfato de cálcio, atingindo a borda da placa de Petri no nono dia de cultivo (Tabela 1). Nos meios contendo fosfato de sódio e fosfato de potássio bibásico o fungo atingiu a borda da placa no $12^{\circ}$ e $15^{\circ}$ dias de cultivo, respectivamente, suportando bom crescimento. A partir do $15^{\circ}$ dia não se verificou diferença estatística no crescimento obtido nessas quatro fontes de fósforo, mas a limitação imposta pelo diâmetro da placa de Petri foi, provavelmente, o fator responsável.

A fonte de fósforo não teve efeito acentuado na esporulação de B. euphorbiae, pois não houve diferença na produção de conídios obtida na presença das diversas fontes, exceto para o ácido fosfórico que promoveu a menor esporulação do fungo (Tabela 2). $\mathrm{O}$ fosfato de potássio monobásico pode ser destacado como a fonte mais favorável, pois estimulou acentuadamente a produção de conídios.

Os resultados obtidos sugeriram que o fosfato de potássio monobásico e o fosfato de cálcio sejam as fontes mais facilmente assimiláveis por $\boldsymbol{B}$. euphorbiae. O fósforo é um elemento indispensável para a síntese de importantes componentes celulares (CARLILE \& WATKINSON, 1994). A adição de fosfatos 
de cálcio, de sódio, de potássio e de amônio a meios com carência de fósforo aumentou acentuadamente o crescimento de A. tenuis Autc Neerg (SINGH \& TANDON, 1967a, b). No entanto, quando cultivada em meios que continham fosfato de potássio, fosfato de potássio tribásico, fosfato de sódio e fosfato de cálcio, A. tenuis não produziu conídios (SINGH \& TANDON, 1967a). B. bassiana produziu dez vezes mais conídios em meio líquido contendo fósforo que no mesmo meio isento de fósforo (THOMAS et al., 1987). V. lecanii não foi influenciado pelas fontes de fósforo, mas o isolado JAB 02 mostrou maior habilidade em utilizar os fosfatos de amônio, de potássio bibásico e de sódio, enquanto para JAB 45 os fosfatos de amônio, de potássio monobásico e bibásico foram as fontes mais favoráveis (WENZEL, 2002).

No ensaio de suplementação do meio de cultivo, o crescimento do fungo foi muito rápido, atingindo a borda da placa de Petri já no nono dia de cultivo em todos os tratamentos, pois o meio mínimo foi elaborado utilizando-se as fontes de carbono, nitrogênio e fósforo que mais favoreceram o crescimento e a esporulação do fungo, ou seja, amido, nitrato de sódio e fosfato de potássio monobásico, respectivamente. Contudo, é possível distinguir que a adição de peptona estimulou o crescimento do fungo (Tabela 1); no terceiro e sexto dias de cultivo promoveu maior crescimento que o obtido no meio mínimo nãosuplementado e não diferiu do observado no meio completo, que contém todos os suplementos. Efeito semelhante foi obtido com a adição de extrato de levedura. A partir do nono, até o último dia de cultivo, não se verificou diferença entre todos os tratamentos, provavelmente pela limitação imposta pelo diâmetro da placa de Petri.

A esporulação obtida em presença de peptona não diferiu da verificada no meio completo e foi maior que a ocorrida no meio mínimo não-suplementado, enquanto a produção de conídios verificada em presença do extrato de levedura e solução de vitaminas se igualou à ocorrida no meio completo e peptona, mas não diferiu da obtida no meio mínimo (Tabela 2).

A suplementação do meio de cultivo com peptona e extrato de levedura estimulou o crescimento e a esporulação do fungo. Peptonas contém mistura de fontes de carbono e nitrogênio que podem ser facilmente utilizáveis por fungos. O extrato de levedura é usado como fonte de nitrogênio para o crescimento de fungos e contém aminoácidos, peptídeos, vitaminas e carboidratos solúveis em água. Possivelmente, alguns desses nutrientes foram utilizados pelo fungo, promovendo o crescimento e a produção de conídios. A adição de vitaminas incrementou a esporulação, sugerindo que sua presença no meio de cultivo possa favorecer o fungo. Todos os fungos precisam de vitaminas, porém, diferem bastante em suas exigências a elas (GARRAWAY \& EVANS, 1984).

Carbono, nitrogênio e fósforo são elementos indispensáveis na nutrição de fungos (CARLILE \& WATKINSON, 1994), mas outros nutrientes como vitaminas e fatores de crescimento são importantes e sua presença no meio pode incrementar o crescimento e a esporulação dos fungos. MARCHIORI et al. (2001) verificaram que o meio sólido preparado com o suco V8 promoveu a produção e favoreceu a viabilidade de conídios de B. euphorbiae, em comparação aos meios feitos com arroz cozido e polpa de abóbora. Segundo os autores, possivelmente esse resultado foi devido à diversidade de nutrientes proporcionada pelos vegetais constituintes do suco.

Para utilização no controle biológico de plantas daninhas, é importante que a produção massal do fungo resulte na maior quantidade possível de conídios, que são as unidades infectivas mais adequadas para a formulação de bioprodutos e o desenvolvimento da doença nas plantas. A produção em larga escala deve, preferencialmente, empregar substratos de baixo custo e fácil obtenção. Os resultados obtidos neste trabalho mostraram que substratos ricos em amido, como grãos de cereais ou determinados resíduos agroindustriais, são os mais indicados para a produção massal de $\boldsymbol{B}$. euphorbiae. Alguns desses substratos podem necessitar de suplementação com nutrientes importantes para o crescimento e a esporulação do fungo. Peptona, extrato de levedura e vitaminas mostraram-se, a priori, adequadas para suplementar tais substratos. Entretanto, novos estudos precisam ser conduzidos para melhor investigar este aspecto, visto que a adição desses ou outros suplementos pode representar um aumento acentuado no custo de produção do bioproduto.

\section{CONCLUSÕES}

O fungo B. euphorbiae utiliza amido, nitrato de sódio e fosfato de potássio monobásico e de cálcio como fontes de carbono, nitrogênio e fósforo, respectivamente, para o crescimento e a esporulação. O desempenho do fungo é incrementado pela suplementação do meio de cultivo com peptona e extrato de levedura, sendo que a adição de vitaminas favorece a produção de conídios.

\section{AGRADECIMENTOS}

Os autores agradecem à Coordenação de Aperfeiçoamento de Pessoal de Nível Superior (CAPES), pela concessão da bolsa de mestrado à primeira autora.

Ciência Rural, v.38, n.7, out, 2008. 


\section{REFERÊNCIAS}

AZEVEDO, J.L.; COSTA, S.O.P. Exercícios práticos de genética. São Paulo: Companhia Editora Nacional e EDUSP, 1973. 288p.

BARBOSA, C.C. et al. Crescimento e esporulação de isolados de Verticillium lecanii sob diferentes condições nutricionais. Pesquisa Agropecuária Brasileira, Brasília, v.37, n.6, p.821$829,2002$.

CARLILE, M.J.; WATKINSON, S.C. The fungi. San Diego: Academic, 1994. 428p.

CHRISTOFFOLETTI, P.J. et al. Resistência de plantas daninhas aos herbicidas. Planta Daninha, Botucatu, v.12, n.1, p.1320, 1994.

COETZEE, J.C.; EICKER, A. The effect of nutricional and environmental factors on the growth and sporulation of a Southern African isolate of Verticillium fungicola. In: INTERNATIONAL CONGRESS ON THE SCIENCE AND CULTIVATION OF EDIBLE FUNGI, 13., 1991, Dublin. Proceedings... Dublin: Balkema, 1991. V.2, p.417-424.

DIAZ, C.G. Comparação entre isolados de Helminthosporium oryzae Breda de Haan quanto a exigências nutricionais e padrão isoenzimático de esterases. 1995. 49f. Dissertação (Mestrado em Fitopatologia) - Escola Superior de Agricultura Luiz de Queiroz, Universidade de São Paulo.

ETEJERE, E.O.; OKOKO, T.A. Seed production, germination and emergence of Euphorbiae heterophylla L. Nigerian Journal of Botany, Ibadan, v.2, p.143-147, 1989.

GARRAWAY, M.O.; EVANS. R.C. Fungal nutrition and physiology. New York: J. Wiley, 1984. 401p.

GAZZIERO, D.L.R.; YORINORI, J.T. Experiência sobre o controle biológico de Euphorbiae heterophylla no Brasil. Jaboticabal: FUNEP- Unesp, 1993. 11p.

MARCHIORI, R. et al. Comparison of culture media for the mass production of Bipolaris euphorbiae and its impact on Euphorbiae heterophylla dry matter accumulation. Summa Phytopathologica, Jaboticabal, v.27, n.4, p.428-432, 2001.

MONTEIRO, A.C. Aspectos fisioecológicos de isolados de fungos entomopatogênicos obtidos na região amazônica (Manaus). 1988. 233f. Tese (Doutorado em Ecologia e Recursos Naturais) - Universidade Federal de São Carlos.
NECHET, K. de L. et al. Bipolaris euphorbiae as a biological control agent for wild poinsettia (Euphorbiae heterophylla): host-specificity and variability in pathogen and host populations. BioControl, Dordrecht, v.51, n.2, p.259-275, 2006

OLIVEIRA, A.S.; SÁ, H.B. de. Taxonomic studies of the Euphorbiaceae JUSS family-I: Euphorbiae heterophylla L. and Euphorbiae cyathophora MURR. Sellowia, Itajaí, v.150, n.40, p.5-31, 1998.

PONTECORVO, G. et al. The genetics of Aspergillus nidulans. Advances in Genetics, San Diego, n.5, p.141-238, 1953.

PRATES, L.G.; FERNANDES, J.M.C. Efeito da temperatura no crescimento micelial de Bipolaris sorokiniana. Fitopatologia Brasileira, Brasília, v.25, n.4, p.661-663, 2000.

SAS INSTITUTE INC. SAS/STAT - User's guide, release 6, 12 TS LEVEL 0020. Cary, 1995.

SILVA, C.M.M. de S.; MELLO, I.S. de. Requisitos nutricionais para o fungo Alternaria alternata. Pesquisa Agropecuária Brasileira, Brasília, v.34, n.3, p.499-503, 1999.

SINGH, B.P.; TANDON, R.N. Phosphorus requirements of certain isolates of Alternaria tenuis Autct. Proceedings of National Academy of Sciences India B, Allahabad, v.37, p.131-134, 1967a.

SINGH, B.P.; TANDON, R.N. Sulfur and phosphorus requirements of Alternaria tenuis Auct. isolated from papaya (Carica papaya L) leaf. Proceedings of National Academy of Sciences India B, Allahabad, v.37, p.199-203, 1967b.

TABER, R.A. et al. A comparative nutritional study of Alternaria raphani, A brassicae, and A brassicicola with special reference to A. raphani. Phytopathology, v.58, p.607-616, 1968.

THOMAS, K.C. et al. Production and properties of Beauveria bassiana conidia cultivated in submerged culture. Canadian Journal of Microbiology, Ottawa, v.33, n.1, p.12-20, 1987.

WENZEL, I.M. Fatores nutricionais e produção em massa de Verticillium lecanii em meios naturais sólidos e líquidos. 2002. 78f. Dissertação (Mestrado em Microbiologia) - Faculdade de Ciências Agrárias e Veterinárias, Universidade Estadual Paulista.

YORINORI, J.T. Biological control of wild poinsettia (Euphorbia heterophylla) with pathogenic fungi. In: DELFOSSE, E.S. (Ed.). SYMPOSIUM ON BIOLOGICAL CONTROL OF WEEDS, 6., 1985, Vancouver, Canada. Proceedings... Vancouver: Vancouver Agriculture Canada, 1985. p.677-681 
O arquivo disponível sofreu correções conforme ERRATA publicada no Volume 38 Número 9 da revista. 